\title{
Pentoxifylline Loaded Floating Microballoons: Design, Development and Characterization
}

\author{
Prashant Malik, Upendra Nagaich, Raj Kaur Malik, and Neha Gulati \\ Department of Pharmaceutics, School of Pharmacy, Bharat Institute of Technology, Meerut 250 103, India \\ Correspondence should be addressed to Prashant Malik; prioxymalik@gmail.com
}

Received 28 January 2013; Revised 16 April 2013; Accepted 16 April 2013

Academic Editor: Danyelle Townsend

Copyright ( 2013 Prashant Malik et al. This is an open access article distributed under the Creative Commons Attribution License, which permits unrestricted use, distribution, and reproduction in any medium, provided the original work is properly cited.

\begin{abstract}
The floating microballoons have been utilized to obtain prolonged and uniform release in the stomach. The objective of the present study involves design, development, and characterization of pentoxifylline loaded floating microballoons to prolong their gastric residence time. Pentoxifylline (trisubstituted xanthine derivative) loaded microballoons were prepared by the solvent evaporation technique using different concentrations of polymers like HPMC K4M and ethyl cellulose (EC) in ethyl alcohol and dichloromethane organic solvent system. Microballoons were characterized for their particle size, surface morphology, production yield, loading efficiency, buoyancy percentage, and in vitro drug release studies. From the characterization it was observed that increases in amount of polymers (HPMC K4M and EC) led to increased particle size, loading efficiency, and buoyancy percentage, and retarded drug release. The particle size, particle yield, loading efficiency, buoyancy percentage and in vitro drug release for optimized formulation (F3) were found to be $104.0 \pm 2.87 \mu \mathrm{m}, 80.89 \pm 2.24 \%, 77.85 \pm 0.61 \%, 77.52 \pm 2.04 \%$, and $82.21 \pm 1.29 \%$, respectively. The data was fitted to different kinetic models to illustrate its anomalous (non-Fickian) diffusion. The in vitro result showed that formulations comprised of varying concentrations of ethyl cellulose in higher proportion exhibited much retarded drug release as compared to formulations comprised of higher proportion of varying concentrations of HPMC K4M.
\end{abstract}

\section{Introduction}

Oral drug delivery systems are essential to optimize both the residence time of the system within the gastrointestinal tract and the release rate of the drug from the system. Various attempts have been made to prolong the residence time of the dosage forms within the stomach [1]. Rapid gastrointestinal transit could result in incomplete drug release from the drug delivery system to absorption window leading to diminished efficacy of the administered dose. Prolonged gastric retention is important in achieving control over the gastric residence time because it helps to maintain the controlled release system in the stomach for a longer time in an expected manner [2]. Floating systems (hydrodynamically controlled systems) are low-density systems which means they are less dense than gastric fluid. These systems have sufficient buoyancy to float over the gastric contents and remain buoyant in the stomach for a prolonged period of time without disturbing the gastric emptying rate. While the formulation is floating on the gastric contents, drug is released slowly from it at a desired rate [3, 4]. For oral sustained release, multiple unit dosage forms (i.e., microballoons) are more beneficial than single unit dosage forms. Because single unit dosage forms have the disadvantage of a release all or nothing emptying process and multiple unit dosage forms have advantages of disperse widely and release drug uniformly along the gastrointestinal tract, which results in more reproducible drug absorption, less dose dumping, and reduced risk of local irritation than the use of single unit dosage form [5-7]. Floating microballoons are gastroretentive drug delivery systems based on a noneffervescent approach. These microballoons are spherical, empty particles without core. These microballoons are characteristically free flowing powders consisting of proteins or synthetic polymers, ideally having a size less than $200 \mu \mathrm{m}$ [8]. Pentoxifylline (PTX), trisubstituted xanthine derivative (3,7-dimethyl-1-(5oxohexyl)-3,7-dihydro- $1 \mathrm{H}$-purine-2,6-dione), is a hemorheologic agent used for the treatment of peripheral arterial disease and intermittent claudication [9]. PTX and its metabolites improve the blood flow by decreasing blood 
TABLE 1: Composition of pentoxifylline loaded microballoons.

\begin{tabular}{|c|c|c|c|c|c|}
\hline Formulation code & HPMC K4M (mg) & Ethyl cellulose (mg) & Solvent ratio (ethanol + dichloromethane) & Tween $80(\%)$ & Drug (mg) \\
\hline F1 & 200 & 200 & $1: 1$ & 0.01 & 200 \\
\hline $\mathrm{F} 2$ & 200 & 400 & $1: 1$ & 0.01 & 200 \\
\hline F3 & 200 & 600 & $1: 1$ & 0.01 & 200 \\
\hline $\mathrm{F} 4$ & 400 & 200 & $1: 1$ & 0.01 & 200 \\
\hline F5 & 600 & 200 & $1: 1$ & 0.01 & 200 \\
\hline F6 & 800 & 200 & $1: 1$ & 0.01 & 200 \\
\hline
\end{tabular}

viscosity. The apparent plasma half-life of the drug and its metabolite is 2-3 hours. On the basis of using PTX as drug of choice in chronic occlusive aterial diseases, it is of a wise candidate drug to be formulated in sustained release oral dosage form [10]. Thus, the aim of the present research work was to design, develope, and characterization of pentoxifylline loaded floating microballoons.

\section{Materials and Methods}

2.1. Materials. Pentoxifylline was obtained as a gift sample from Bakul Pharma Pvt. Ltd. Mumbai, India. Ethyl cellulose, hydroxypropyl methylcellulose (HPMC K4M), and Tween 80 were purchased from Central Drug House $(\mathrm{CDH})$, New Delhi, India. All other solvents and chemicals were of analytical grade.

\subsection{Methods}

2.2.1. Preparation of Microballoons. Microballoons were prepared by the solvent evaporation technique [11]. Pentoxifylline, HPMC K4M, and ethyl cellulose were dissolved in a mixture of ethanol and dichloromethane at room temperature. These were poured into $250 \mathrm{~mL}$ of water containing $0.01 \%$ Tween 80 maintained at a temperature of $30-40^{\circ} \mathrm{C}$ and consequently stirred at ranging agitation speed to allow the volatile solvent to evaporate. The formulated microballoons were filtered, washed with distilled water, and dried at $40^{\circ} \mathrm{C}$. The composition of various formulations is shown in Table 1.

\subsubsection{Characterization of Microballoons}

Particle Size. The size of microballoons of each formulation was determined using a microscope fitted with an ocular micrometer, and stage micrometer and average particle size was determined [12].

Surface Morphology. The surface morphology of microballoons was examined by scanning electron microscopy (Jeol JSM-1600, Tokyo, Japan) operated at $15 \mathrm{kV}$ on samples gold sputtered at $10 \mathrm{~mA}$, under argon at low pressure.

Determination of Production Yield. The prepared microballoons were collected and weighed. The weight of microballoons was divided by the total weight of all the nonvolatile components that were used for the preparation of the microballoons and multiplied by 100 gives the \% yield of microballoons [5] as follows:

$$
\begin{aligned}
\% \text { Yield }= & (\text { weight of microballoons collected }) \\
& \times(\text { weight of all nonvolatile components } \\
& \text { used for the preparation })^{-1} \times 100 .
\end{aligned}
$$

Percentage Loading Efficiency. To determine loading efficiency, microballoons were taken, thoroughly triturated, and suspended in a minimal amount of alcohol. The suspension was suitably diluted with water and filtered to separate shell fragments. The estimation of drug was carried out using UV spectrophotometer (UV-VIS double beam spectrophotometer 2201, Systronics) at $272 \mathrm{~nm} \lambda_{\max }$ [6]. The percentage loading efficiency was calculated as follows:

$$
\begin{aligned}
& \text { Loading efficiency (\%) } \\
& \qquad=\frac{\text { amount of drug actually present }}{\text { theoretical drug load expected }} \times 100 \text {. }
\end{aligned}
$$

Buoyancy Percentage. The buoyancy test of the microballoons was carried out using USP II (paddle type) dissolution apparatus (DS 8000, LABINDIA). Dissolution test solution simulated gastric fluid (SGF) containing Tween $80(0.02 \%$ $\mathrm{v} / \mathrm{v}$ ) was used as a dispersion medium to simulate gastric fluid. The microballoons were spread over the surface of the SGF, pH $1.2\left(900 \mathrm{~mL}, 37 \pm 0.5^{\circ} \mathrm{C}\right)$, which was agitated by a paddle rotated at $100 \mathrm{rpm}$ for $12 \mathrm{~h}$. After agitation for a previously determined interval, the microballoons that were floating and the ones that settled to the bottom of the flask were recovered separately [2]. After drying, the fraction of the microballoons was weighed. The \% buoyancy of the microballoons was calculated by the following formula:

$$
\begin{aligned}
& \% \text { Buoyancy } \\
& =\frac{\text { weight of floating microballoons after drying }}{\text { weight of floating }+ \text { settled microballoons after drying }} \\
& \quad \times 100 .
\end{aligned}
$$

In Vitro Drug Release Studies. The in vitro drug release from microballoons was determined using USP II dissolution apparatus. The dissolution test was performed 
TABLE 2: Characterization of pentoxifylline loaded microballoons.

\begin{tabular}{|c|c|c|c|c|c|c|}
\hline \multirow{2}{*}{ Parameters } & \multicolumn{6}{|c|}{ Formulation code } \\
\hline & $\mathrm{F} 1$ & F2 & F3 & $\mathrm{F} 4$ & F5 & F6 \\
\hline Particle size $(\mu \mathrm{m})^{\mathrm{a}}$ & $74.63 \pm 1.04$ & $85.18 \pm 3.12$ & $104.0 \pm 2.87$ & $82.96 \pm 2.13$ & $99.32 \pm 1.45$ & $110.4 \pm 2.94$ \\
\hline Production yield $(\%)^{\mathrm{a}}$ & $75.76 \pm 1.54$ & $78.13 \pm 1.21$ & $80.89 \pm 2.24$ & $76.79 \pm 1.38$ & $74.66 \pm 2.61$ & $72.57 \pm 1.85$ \\
\hline Incorporation efficiency $(\%)^{\mathrm{a}}$ & $75.5 \pm 1.82$ & $76.36 \pm 1.27$ & $77.85 \pm 0.61$ & $76.22 \pm 0.82$ & $77.29 \pm 0.12$ & $77.66 \pm 1.35$ \\
\hline Buoyancy $(\%)^{\mathrm{a}}$ & $72.43 \pm 0.21$ & $74.28 \pm 1.82$ & $77.52 \pm 2.04$ & $73.64 \pm 1.73$ & $76.24 \pm 0.82$ & $78.19 \pm 0.63$ \\
\hline In vitro drug release $(\%)^{\mathrm{a}}$ & $96.81 \pm 0.16$ & $88.84 \pm 0.46$ & $82.21 \pm 1.29$ & $93.13 \pm 1.48$ & $90.16 \pm 0.98$ & $87.09 \pm 1.73$ \\
\hline
\end{tabular}

${ }^{a}$ Each value indicates the mean \pm SD $(n=3)$.

using $0.1 \mathrm{~N} \mathrm{HCl}(\mathrm{pH} 1.2)$ as dissolution fluid $(900 \mathrm{~mL})$ maintained at $37 \pm 0.5^{\circ} \mathrm{C}$ at $100 \mathrm{rpm}$. The samples $(5 \mathrm{~mL})$ of the solution were withdrawn from the dissolution apparatus for $12 \mathrm{~h}$, and the samples were replaced with fresh dissolution medium each time to maintain the sink condition. Withdrawn samples were analyzed using UV-VIS double beam spectrophotometer at $272 \mathrm{~nm}$ against suitably constructed calibration curve. All measurements were carried out in triplicate, and average values were plotted [11].

Statistical Analysis. Two-way analysis of variance (ANOVA) was applied to check significant differences in drug release from different formulations. Differences were considered to be statistically significant at $P<0.05$ [6].

Drug Release Kinetics. Data obtained from in vitro release study was fitted into various kinetic equations. The kinetic models used were zero order (cumulative percentage of drug release versus time), first order (log cumulative percentage of drug remaining versus time), the Higuchi model (cumulative percentage of drug release versus square root of time), and Korsmeyer-Peppas (log cumulative percent drug release versus log of time) [13]. Regression $\left(r^{2}\right)$ values were calculated for the linear curves obtained by regression analysis.

\section{Results and Discussion}

3.1. Preparation of Microballoons. Preparation of pentoxifylline loaded floating microballoons was done by using HPMC K4M and ethyl cellulose as sustained release polymers by the solvent evaporation technique. Ethanol and dichloromethane were used as solvents to keep both polymers and drug in solution. The solution was poured with the help of syringe into $250 \mathrm{~mL}$ water containing $0.01 \%$ Tween 80 maintained at a temperature of $30-40^{\circ} \mathrm{C}$ and subsequently stirred at ranging agitation speed to allow the volatile solvent to evaporate. The formulations F1, F2, and F3 were formulated by varying the concentration of ethyl cellulose, and formulations F4, F5, and F6 formulated by varying the concentration of HPMC K4M. Microballoons with higher concentration of ethyl cellulose gave much retarded drug release than higher concentration of HPMC K4M.

3.2. Particle Size. From the result of this study, the average particle size of microballoons were found to be $74.63 \pm$ $1.04,85.18 \pm 3.12$, and $104.0 \pm 2.87$ for F1, F2, and F3 formulations and $82.96 \pm 2.13,99.32 \pm 1.45$, and $110.4 \pm 2.94$

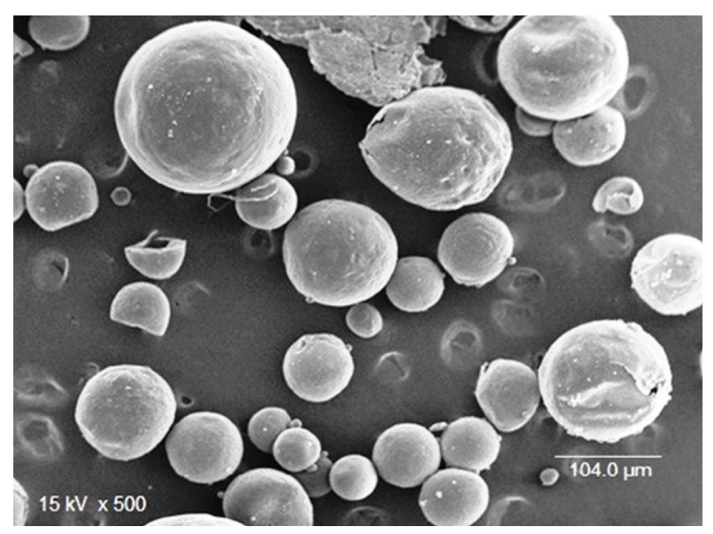

FIGURE 1: Scanning electron microphotograph of floating microballoons.

for F4, F5, and F6 formulations, respectively. The particle size increased with increasing polymers concentration. This is due to the increase in viscosity of the solution and the decrease in stirring efficiency. Also with increasing polymer concentration, the hardening time of the microballoons was shortened. Therefore, a shorter time was provided for the breakup of droplets, and larger microballoons were formed [14].

3.3. Surface Morphology. The scanning electron microphotograph showed that the developed floating microballoons were spherical with porous surface which facilitate diffusion of drug as shown in Figure 1.

3.4. Production Yield. Production yields were found to be $75.76 \pm 1.54,78.13 \pm 1.21,80.89 \pm 2.24,76.79 \pm 1.38,74.66 \pm 2.61$, and $72.57 \pm 1.85$ for F1, F2, F3, F4, F5, and F6 formulations, respectively, as shown in Table 2.

3.5. Percentage Loading Efficiency. The percentage loading efficiencies were found to be $75.5 \pm 1.82,76.36 \pm 1.27,77.85 \pm$ $0.61,76.22 \pm 0.82,77.29 \pm 0.12$, and $77.66 \pm 1.35 \%$ for $\mathrm{F} 1, \mathrm{~F} 2$, F3, F4, F5, and F6 formulations, respectively.

3.6. Buoyancy Percentage. The buoyancy percentage for all batches was almost above $70 \%$, which was studied for $12 \mathrm{~h}$. The highest percentage was obtained with formulation F6. Average buoyancies in percentage were found to be in 
TABLE 3: Release kinetic data obtained from different plots of models.

\begin{tabular}{lcccccccc}
\hline \multirow{2}{*}{ Formulation code } & \multicolumn{2}{c}{ Zero order } & \multicolumn{2}{c}{ First order } & \multicolumn{2}{c}{ Higuchi } & \multicolumn{2}{c}{ Korsmeyer-Peppas } \\
& $K$ & $r^{2}$ & $K$ & $r^{2}$ & $K$ & $r^{2}$ & $n$ \\
\hline F1 & 7.918 & 0.896 & 0.104 & 0.534 & 30.31 & 0.985 & 0.776 \\
F2 & 7.341 & 0.922 & 0.106 & 0.569 & 27.79 & 0.991 & 0.799 & 0.522 \\
F3 & 7.014 & 0.956 & 0.112 & 0.639 & 26.03 & 0.987 & 0.842 & 0.565 \\
F4 & 7.570 & 0.915 & 0.104 & 0.547 & 28.77 & 0.991 & 0.776 & 0.533 \\
F5 & 7.426 & 0.915 & 0.105 & 0.557 & 28.20 & 0.990 & 0.788 & 0.549 \\
F6 & 7.296 & 0.925 & 0.109 & 0.586 & 27.56 & 0.989 & 0.825 & 0.592 \\
\hline
\end{tabular}

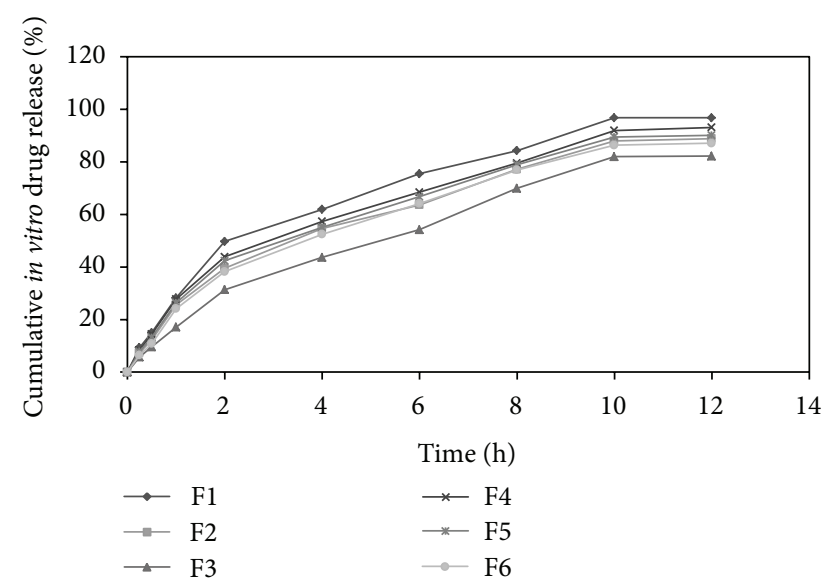

FIGURE 2: Release profile of pentoxifylline from microballoons for all formulations.

the range of $72.43 \pm 0.21 \%$ to $78.19 \pm 0.63 \%$ for F1 to F6 formulations. In general, with the increase in the amount of polymers, there was an increase in the buoyancy percentage. The increase in the buoyancy percentage may be attributed to air and gel-forming polymer HPMC K4M which caused swelling because of increased amount of the polymers present [15].

3.7. In Vitro Drug Release Studies. The in vitro drug release of formulations F1, F2, F3, F4, F5, and F6 was found to be $96.81 \pm 0.16,88.84 \pm 0.46,82.21 \pm 1.29,93.13 \pm 1.48,90.16 \pm 0.98$, and $87.09 \pm 1.73$ in $12 \mathrm{~h}$, respectively. Results indicate that proportion of polymers in formulation was the key factor governing the release of drug from microballoons. As the concentration of polymer increased, there was an increase in diffusional path length. This may decrease the overall drug release from the polymer matrix. Formulations comprised of ethyl cellulose in higher proportion exhibited much retarded drug release as compared to formulations comprised of HPMC K4M in higher proportion [16]. The release profile of pentoxifylline from microballoons for all formulations was shown in Figure 2. The release profile of pentoxifylline from microballoons containing varying concentrations of ethyl cellulose and HPMC K4M was shown in Figures 3 and 4, respectively.

3.8. Statistical Analysis. Two-way analysis of variance (ANOVA) was applied to check significant differences in drug release from different formulations containing different

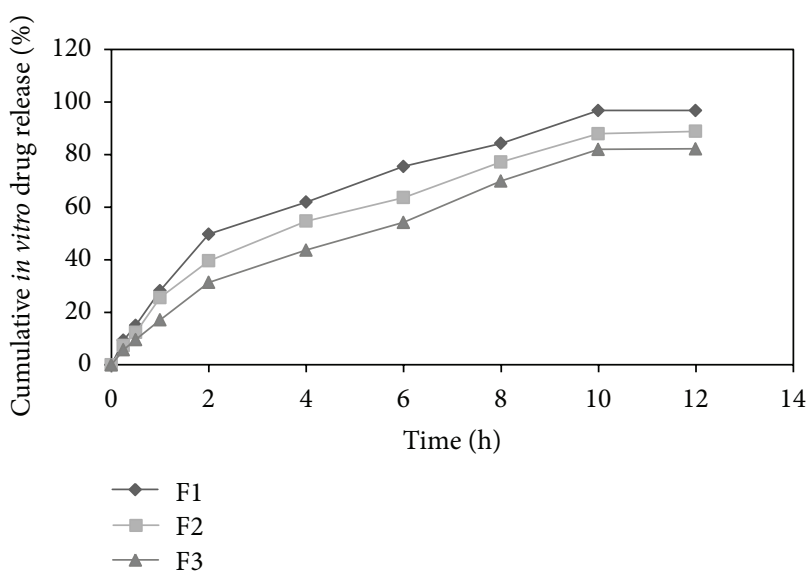

FIGURE 3: Release profile of pentoxifylline from microballoons containing varying concentrations of ethyl cellulose.

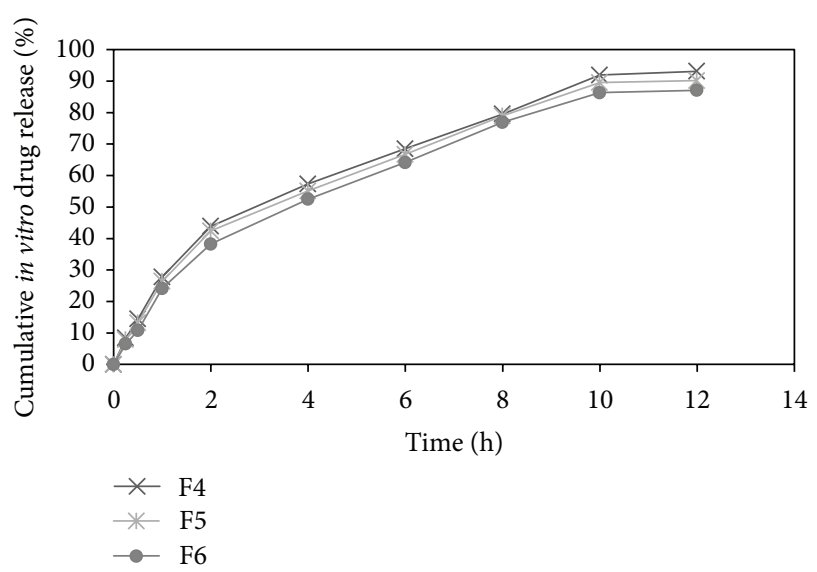

FIGURE 4: Release profile of pentoxifylline from microballoons containing varying concentrations of HPMC K4M.

concentrations of HPMC K4M and ethyl cellulose. On increasing the amount of polymers, a significant decrease $(P<0.05)$ was obtained in the cumulative drug release.

3.9. Drug Release Kinetics. The kinetics and mechanism of drug release were determined using zero order, and first order, Higuchi's model, and further analysis was performed using Korsmeyer-Peppas equation. All formulations were found to be following Higuchi's model as the plot showed high linearity $\left(r^{2}=0.985\right.$ to 0.991$)$ as shown in Table 3 . 
This equation indicates that the cumulative amount of drug release is proportional to the square root of time for diffusional release of drug from the formulation. The calculated " $n$ " values from the power law equation (KorsmeyerPeppas equation) for drug release profiles were between 0.776 and 0.842 , suggesting that drug release mechanism from formulations followed the non-Fickian (anomalous) transport mechanism, which may indicate that diffusion was predominant mechanism of drug release [17]. The release kinetic data obtained from different plots of models for all formulations are given in Table 3.

\section{Conclusion}

Floating microballoons of pentoxifylline were prepared by the solvent evaporation technique using different concentrations of polymers like HPMC K4M and ethyl cellulose (EC) dispersed in ethyl alcohol and dichloromethane as a solvent system. Prepared floating microballoons showed significant floating ability, good buoyancy, and sustained drug release. In vitro drug release of microballoons was influenced by polymers concentration. From the percentage loading efficiency and in vitro drug release studies, it was observed that F3 formulation exhibits greater drug loading efficiency and sustained release behavior. On fixing the in vitro drug release data of optimized formulation to various kinetic models, it was found that it exhibits the Higuchi order of kinetics followed by zero order and first order. The formulation undergoes anomalous (non-Fickian) diffusion, which indicates that the drug release rate was controlled by swelling, erosion, and diffusion from microballoons. Thus, pentoxifylline loaded floating microballoons can prove to be potential pharmaceutical dosage form for prolonging the gastric retention time of dosage form.

\section{Acknowledgments}

The authors are thankful to Bakul Pharma Pvt. Ltd., Mumbai, India, for providing the pentoxifylline as a gift sample. The authors are also thankful to the Board of Trustees, Bharat Institute of Technology, Meerut, India, for providing necessary facilities to carry out this research work.

\section{References}

[1] S. Ramachandran, S. M. Shaheedha, G. Thirumurugan, and M. D. Dhanaraju, "Floating controlled drug delivery system of famotidine loaded hollow microspheres (microballoons) in the stomach," Current Drug Delivery, vol. 7, no. 1, pp. 93-97, 2010.

[2] N. K. Gorde, H. A. Pawar, N. Khutle, and Y. Chaudhari, "Formulation development and optimization of floating microballoons for oral delivery of domperidone," International Journal of Pharmaceutical and Phytopharmacological Research, vol. 2, no. 2, pp. 101-108, 2012.

[3] A. V. Mayavanshi and S. S. Gajjar, "Floating drug delivery systems to increase gastric retention of drugs: a review," Research Journal of Pharmacy and Technology, vol. 1, pp. 345-348, 2008.

[4] M. Jelvehgari, M. Maghsoodi, and H. Nemati, "Development of theophylline floating microballoons using cellulose acetate butyrate and/or Eudragit RL 100 polymers with different permeability characteristics," Research in Pharmaceutical Sciences, vol. 5, no. 1, pp. 29-39, 2010.

[5] A. K. Chaturvedi, A. Verma, A. Singh, and A. Kumar, "Formulation and characterization of microballoons of norfloxacin," Journal of Drug Delivery and Therapeutics, vol. 1, no. 2, pp. 21-26, 2011.

[6] P. Bhardwaj, H. Chaurasia, D. Chaurasia, S. K. Prajapati, and S. Singh, "Formulation and in-vitro evaluation of floating microballoons of indomethacin," Acta Poloniae Pharmaceutica, vol. 67, no. 3, pp. 291-298, 2010.

[7] A. Patel, S. Ray, and R. S. Thakur, "In vitro evaluation and optimization of controlled release Floating Drug Delivery System of metformin hydrochloride," Daru, vol. 14, no. 2, pp. 57-64, 2006.

[8] A. Chandra, S. K. Prajapati, S. K. Jain, and S. Singh, "Development and in-vitro/in-vivo evaluation of floating microballoons of flurbiprofen," Journal of Pharmaceutical Science and Technology, vol. 3, pp. 725-734, 2011.

[9] H. R. Dettelbach and D. M. Aviado, "Clinical pharmacology of pentoxifylline with special reference to its hemorrheologic effect for the treatment of intermittent claudication," Journal of Clinical Pharmacology, vol. 25, no. 1, pp. 8-26, 1985.

[10] A. H. Hussain and Y. I. Khalil, "Some variables affecting the formulation of pentoxifylline (PTX) as a solid sustained release dosage form," Iraqi Journal of Pharmaceutical Sciences, vol. 17, no. 1, pp. 1-6, 2008.

[11] A. Yadav and D. K. Jain, "Gastroretentive microballoons of metformin: formulation development and characterization," Journal of Advanced Pharmaceutical Technology and Research, vol. 2, no. 1, pp. 51-55, 2011.

[12] A. K. Jain, C. P. Jain, K. Gaur, A. Kakde, M. Meena, and R. K. Nema, "Effect of natural biodegradable and synthetic polymer for gastric disease by floating microspheres," Continental Journal Pharmaceutical Sciences, vol. 3, pp. 1-6, 2009.

[13] T. Higuchi, "Mechanism of sustained action medication. Theoretical analysis of rate of release of solid drugs dispersed in solid matrices," Journal of Pharmaceutical Sciences, vol. 52, pp. 11451149, 1963.

[14] M. Maghsoodi, E. Hemati, B. Qadermazi, and Z. Yari, "Hollow microspheres for gastroretentive floating-pulsatile drug delivery: preparation and in vitro evaluation," Advanced Pharmaceutical Bulletin, vol. 1, no. 2, pp. 55-61, 2011.

[15] M. K. Deepa and M. Karthikeyan, "Cefpodoxime proxetil floating microspheres: formulation and in vitro evaluation," Iranian Journal of Pharmaceutical Sciences, vol. 5, no. 2, pp. 69-72, 2009.

[16] A. K. Sharma, R. K. Keservani, S. C. Dadarwal, Y. L. Choudhary, and S. Ramteke, "Formulation and in vitro characterization of cefpodoxime proxetil gastroretentive microballoons," DARU, vol. 19, no. 1, pp. 33-40, 2011.

[17] S. Dash, P. N. Murthy, L. Nath, and P. Chowdhury, "Kinetic modeling on drug release from controlled drug delivery systems," Acta Poloniae Pharmaceutica, vol. 67, no. 3, pp. 217-223, 2010. 

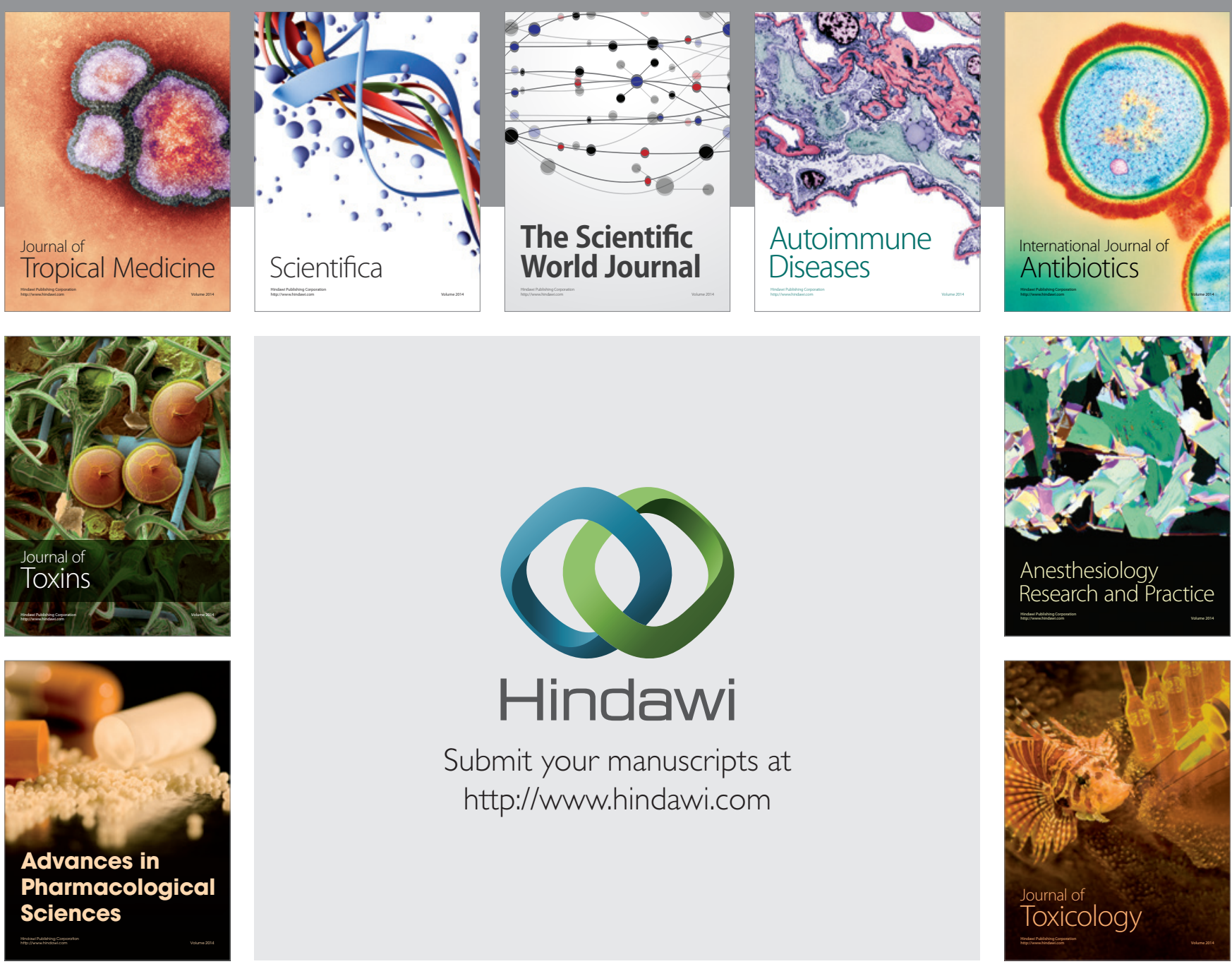

\section{Hindawi}

Submit your manuscripts at

http://www.hindawi.com
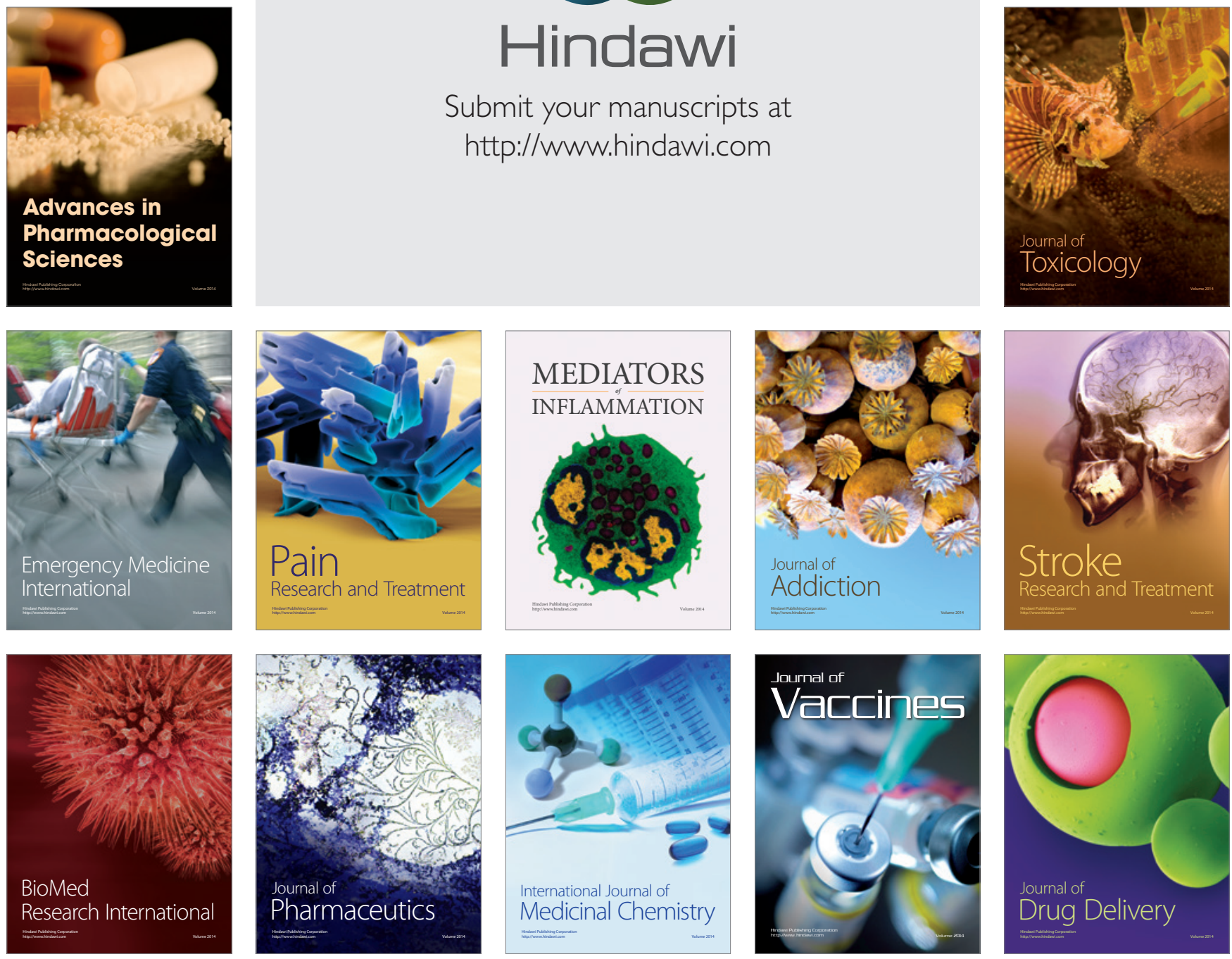